\title{
On the relation between DC current locations and an EUV bright point: A case study
}

\author{
J. C. Santos ${ }^{1,2}$, J. Büchner ${ }^{1}$, M. S. Madjarska ${ }^{1}$, and M. V. Alves ${ }^{2}$
}

\author{
1.Max-Planck-Institut für Sonnensystemforschung, Max-Planck-Str. 2, 37191 Katlenburg-Lindau, Germany \\ e-mail: santos@mps.mpg.de \\ 2.Instituto Nacional de Pesquisas Espaciais, Av. dos Astronautas 1758, Jd. da Granja, 12227-010 São José dos Campos, \\ São Paulo, Brazil \\ e-mail: virginia@plasma.inpe.br
}

Received 14 April 2008 / Accepted 20 August 2008

\section{ABSTRACT}

\begin{abstract}
Context. Motion of the photospheric plasma forces the footpoints of magnetic flux tubes to move. This can give rise to electric currents in the solar atmosphere. The dissipation of these electric currents and the consequent heating of the solar plasma may be responsible for the formation of Extreme-UltraViolet (EUV) and X-ray bright points. Earlier bright point models usually consider either the emergence or the canceling of photospheric magnetic features as being responsible for reconnection causing the bright point. Aims. We investigate the consequences of different patterns of horizontal photospheric plasma motion for the generation of electric currents in the solar atmosphere and locate them with respect to an observed EUV bright point. The goal is to find out whether these currents might be responsible for the heating of bright points.

Methods. To perform this study we use a "data driven" three dimensional magnetohydrodynamic model. The model solves an appropriate set of magnetohydrodynamic equations and uses, as initial condition, the magnetic field extrapolated from the line-of-sight component of the photospheric magnetic field observed by MDI/SoHO and the height-stratified, equilibrium density and temperature of the solar corona. We apply different patterns of horizontal photospheric plasma motion, derived from the temporal evolution of the photospheric magnetic structures in the course of the bright point lifetime, as boundary conditions of the model.

Results. All applied patterns of horizontal photospheric plasma motion (shearing, convergence and fragmentation) lead to the formation of electric currents in the chromosphere, transition region and corona. Currents do not develop everywhere in the region where the motion is applied but in specific places where the magnetic field connectivity changes significantly. An important result is that the position where the electric currents develop is independent of the motion pattern used as boundary condition of the model. A comparison with data obtained by TRACE in the $1550 \AA$ channel and by the EIT in the $195 \AA$ channel shows that the region where the strongest current concentrations are formed coincides with the region where the EUV bright point appears.
\end{abstract}

Key words. Sun: atmosphere - Sun: magnetic fields - Sun: activity - magnetohydrodynamics (MHD) - methods: numerical

\section{Introduction}

Extreme-UltraViolet (EUV) and X-ray Bright Points (BPs) (Golub 1980; Webb 1986; Habbal 1992) provide a specific example of solar corona heating. The two main groups of models used to explain coronal heating attribute the heating of the solar plasma either to the dissipation of waves (Alfvén 1947) or electric currents (Parker 1972) in the solar atmosphere. In both cases the energy source is kinetic energy associated with photospheric plasma motion.

In this sense, an important feature that can help to understand the nature of the heating is the fact that BPs often are associated with moving bipolar magnetic features. Previous models (Tur \& Priest 1976; Priest et al. 1994; Parnell et al. 1994; Longcope 1998) already took into account this characteristics to try to explain BPs. However, the models consider that the moving bipolar magnetic features have a single motion pattern (emerging or canceling) only and they also do not consider plasma motion through regions of strongly diverging magnetic fields. During the evolution of one BP, Brown et al. (2001) found different patterns of motion of the magnetic structures in the solar photosphere. The patterns of motion, coalescence, fragmentation, rotation and translation, were associated with different stages of the evolution of the BP: pre-brightening stage, the initial brightening, the sigmoid phase and the $\pi$-phase, respectively. Such motions could generate currents that cannot be easily dissipated in the corona through conventional joule heating (Parker 1972), but by means of an anomalous resistivity (Birn \& Priest 2007; Büchner \& Elkina 2005, 2006).

The development of electric currents due to horizontal plasma motion in the photosphere and chromosphere was already investigated by Büchner et al. (2004a,b), Büchner (2006) and Santos \& Büchner (2007) for different BP cases. They showed that horizontal plasma motion in the photosphere and chromosphere causes the formation of electric currents below the corona at the position of the EUV BP. It was suggested that the enhanced current flow can make the current sheet resistive and allows stress relaxation by current dissipation which powers the BP. However, these models considered a single motion pattern for the magnetic structures associated with the BP.

Here we use a 'data driven' three-dimensional (3D) magnetohydrodynamic (MHD) model (Büchner et al. 2004a) to study the evolution of plasma and magnetic field in an EUV BP region observed on 2006 January 19 in dependence on different kinds of photospheric plasma motion. A preliminary study of the evolution of electric currents in the solar atmosphere due to 


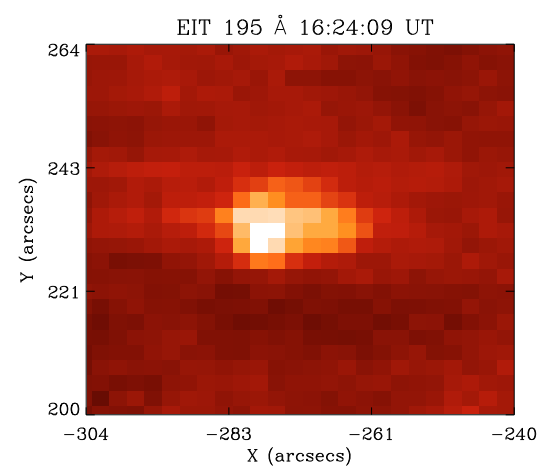

TRACF $1550 \AA 16 \cdot 21: 51$ UT

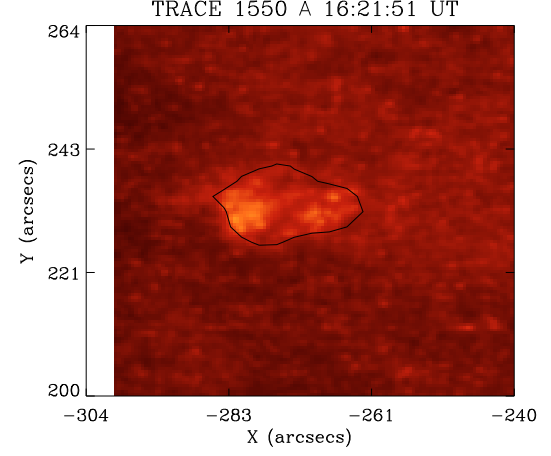

MDI Magnetogram

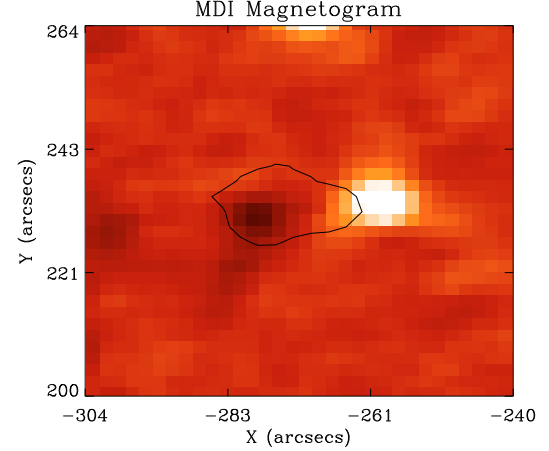

EIT $195 \AA$ \& 17:00:09 UT

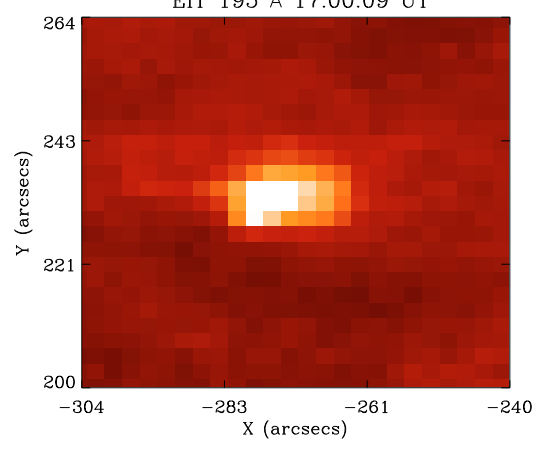

TRACE $1550 \AA$ 17:08:56 UT

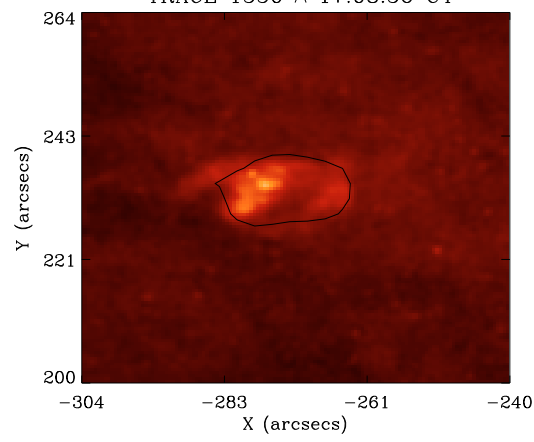

MDI Magnetogram

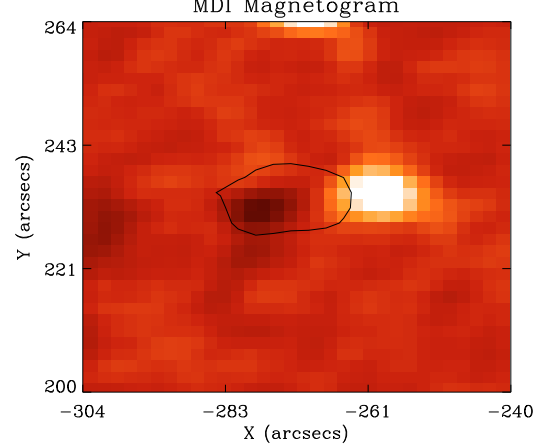

EIT $195 \AA$ 17:36:10 UT

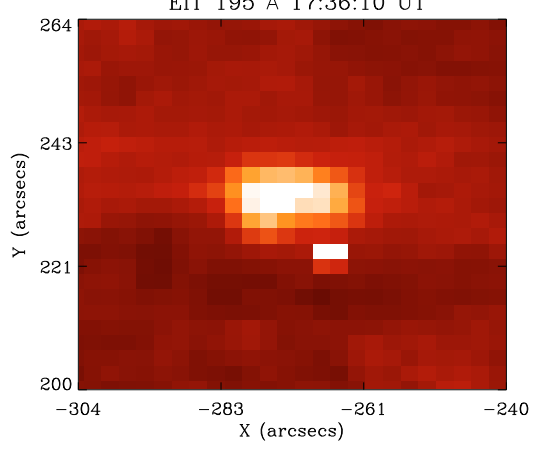

TRACE $1550 \AA$ 17:29:41 UT

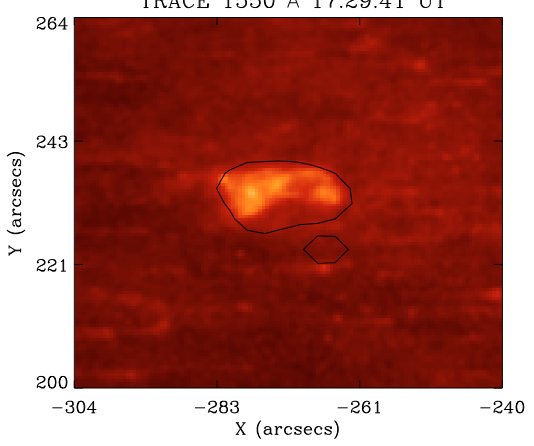

MDI Magnetogram

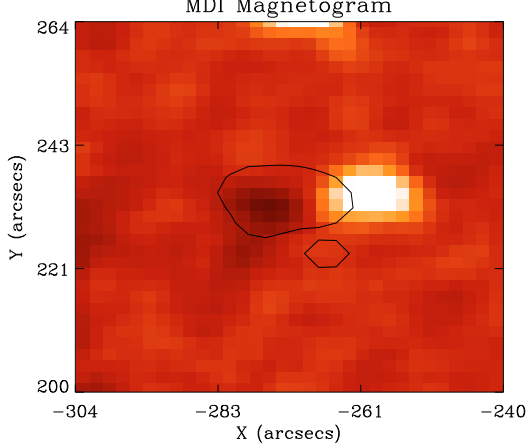

Fig. 1. Evolution of the EUV BP as seen by EIT/SoHO (top panels) and TRACE (middle panels), together with the LOS component of the photospheric magnetic field measured by MDI/SoHO (bottom panels). The images were obtained at three different instants of time: at around 16:30 UT (left column), 17:00 UT (central column) and 17:30 UT (right column). The contour line shows the position of the EUV BP as seen in the EIT $195 \AA$ passband.

a specific pattern of motion for this BP case was given in Santos \& Büchner (2007) for a short part of the BP lifetime, i.e. from 16:00 UT until 16:30 UT. Here we extend their work by considering two additional patterns of motion taking place during the ongoing evolution of the BP. The velocity patterns are derived from the evolution of the photospheric magnetic features associated with the BP. They are applied as boundary condition to the model to simulate the evolution of the system between 16:30 UT and 17:30 UT. The goal is to determine the differences, if they exist, in the distribution of electric currents generated by the different motion patterns used to drive the evolution of the system. The observations are described in Sect. 2. The results obtained for the parallel and perpendicular currents using different velocity fields are presented in Sect. 3. Our main conclusions are given in Sect. 4.

\section{Observational data}

We apply the model to an EUV BP observed on 2006 January 19 by the Extreme-ultraviolet Imaging Telescope (EIT) in the Fe XII $195 \AA$ passband, the Transition Region And Coronal
Explorer (TRACE) in the $1550 \AA$ passband (dominated by the C IV $1548 \AA$ line and continuum emission) and the Michelson Doppler Imager (MDI). The BP appears in the EIT $195 \AA$ images as a feature of diffused enhanced emission with a bright kernel which evolves in time (Fig. 1, top). Due to the relatively low spatial resolution of the EIT images (2.62"/pixel), the fine structure of the BP cannot be resolved. Even though, the general evolution of the feature which has a size of $30^{\prime \prime} \times 20^{\prime \prime}$ can be tracked. The TRACE $1550 \AA$ images $\left(0.5^{\prime \prime} /\right.$ pixel) clearly show the BP as composed of numerous small-scale bright points (diameter comparable with the instrument resolution) believed to represent a cross-section of the BP loops (Fig. 1, middle). MDI provided LOS magnetograms at half resolution $(2 \times 2$ pixels binned images resulting in 1.99"/pixel, bottom). The BP is associated with a magnetic bipolar region (Brown et al. 2001) with the bright kernel overlying the negative polarity (Fig. 1, bottom). The alignment of the imager (EIT) and magnetogram data was done through the header pointing information.

The velocity responsible for the evolution of the photospheric magnetic structures associated with the EUV BP is obtained using the local correlation tracking (LCT) technique 
a

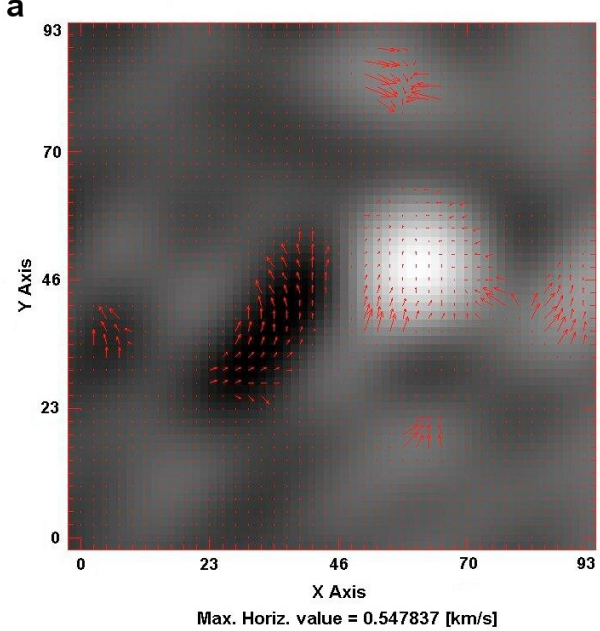

$B_{2}[G]$
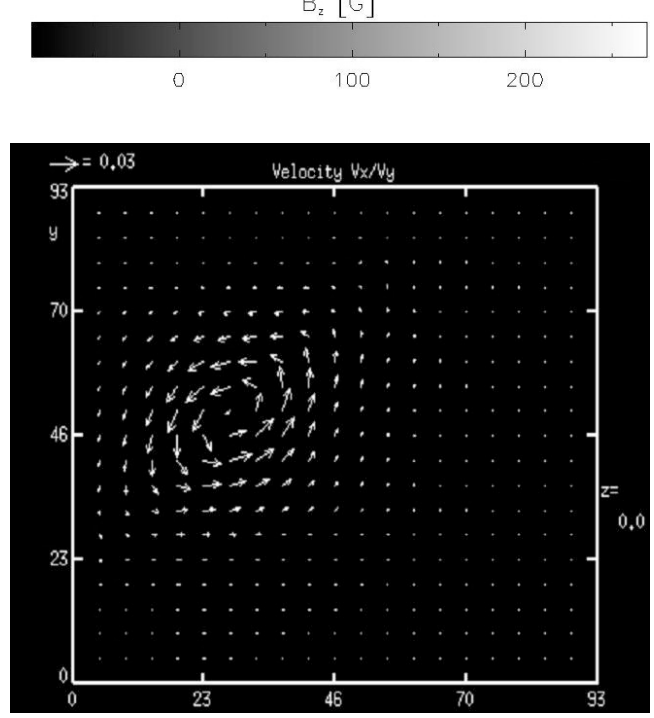

b

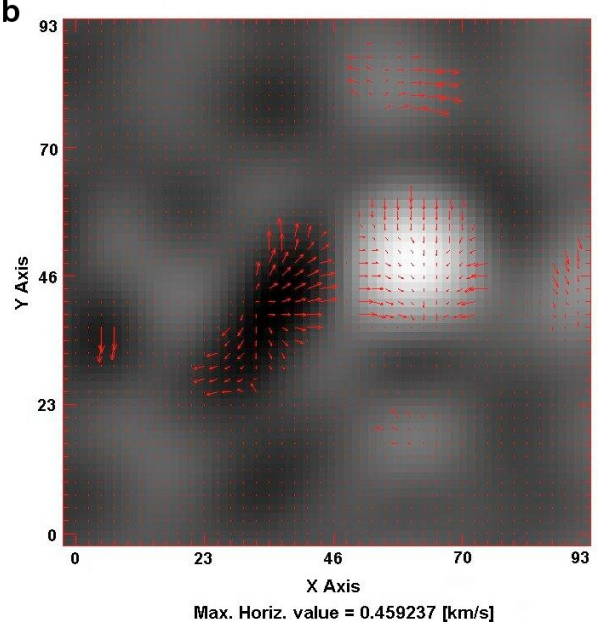

Max. Horiz. value $=0.459237[\mathrm{~km} / \mathrm{s}]$

$B_{2}[G]$
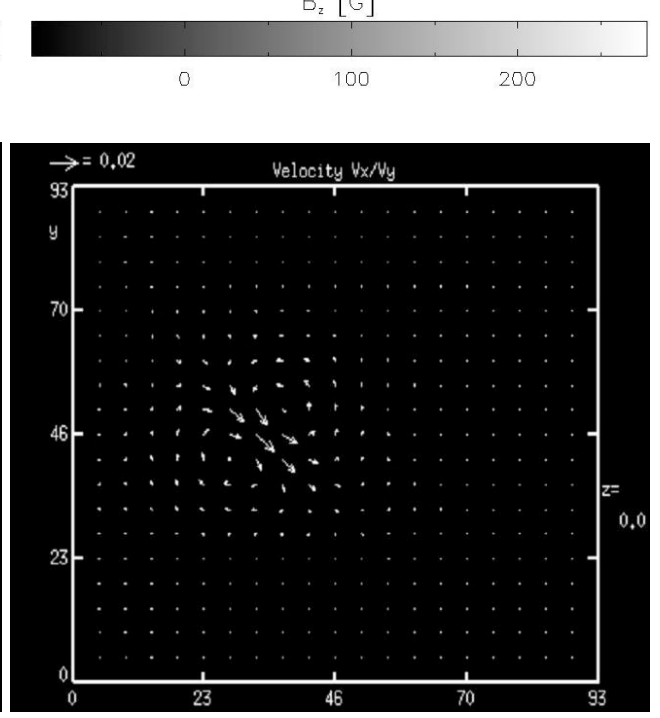

Fig. 2. Horizontal velocity obtained using LCT technique applied to the filtered photospheric magnetic field in the interval a) 16:30 UT-17:00 UT and b) 17:00 UT-17:30 UT. The arrows show the horizontal velocity, while the gray scale shows the filtered LOS component of the photospheric magnetic field. The $X$ and $Y$ axis are in terms of the characteristic length scale $\left(L_{0}=\right.$ $\left.5 \times 10^{5} \mathrm{~m}\right)$ and they cover the same area displayed in the MDI image of Fig. 1.

Fig. 3. Horizontal velocity used as boundary condition of the model to approximate the velocity pattern obtained for the interval 16:30 UT17:00 UT (left panel) and 17:00 UT17:30 UT (right panel). The arrows show the horizontal velocity. The maximum value of the horizontal velocity is given on the top left of each panel in terms of the Alfvén velocity $\left(v_{A}=\right.$ $5 \times 10^{4} \mathrm{~m} / \mathrm{s}$ ). The $X$ and $Y$ axis are in terms of the characteristic length scale $\left(L_{0}=5 \times 10^{5} \mathrm{~m}\right)$ and they cover the same area displayed in the MDI image of Fig. 1. The $\mathrm{V} x / \mathrm{V} y$ over the panels means that the velocity vectors in $x-y$ plane are shown.
(November \& Simon 1988). After the LOS component of the photospheric magnetic field is filtered, using a Fourier filter to select the first eight modes of interest, the LCT technique is applied to the filtered magnetograms separated by a time interval of approximately $30 \mathrm{~min}$, covering the interval between 16:30 UT and 17:30 UT. As a result, two different velocity patterns are obtained, which are shown in Fig. 2. The horizontal motion around the positive magnetic field concentration are considered an effect of the emergence of magnetic flux in that region interpreted by LCT as a horizontal velocity pattern (Démoulin \& Berger 2003), and are discarded. We focus on the horizontal motion derived from the displacement around the negative magnetic field concentration. In the simulation model this motion is approximated by using a combination of vortices of velocity (Fig. 3). The first velocity pattern (Fig. 2a) is approximated using a vortex that moves the negative polarity toward the positive polarity. The second velocity pattern (Fig. 2b) is approximated by combining two small vortices that break the negative polarity apart, moving part of it away from the positive polarity and the other part toward the positive polarity. The combination of the vortices in the second velocity pattern enhances the flow in the region between the vortices and makes the full vortical structure visually disappears. The $\mathrm{V} x / \mathrm{V} y$ over the panels in Fig. 3 means that the velocity vectors in $x-y$ plane are shown. The velocity pattern responsible for the evolution between 16:00 UT and 16:30 UT is presented in Fig. 1 of Santos \& Büchner (2007). It is approximated using a vortex (see Fig. 2 of Santos \& Büchner 2007) that moves the negative polarity away from the positive polarity. The three patterns of motion are responsible for the evolution of the photospheric plasma and magnetic field for the interval between 16:00 UT and 17:30 UT. The initial threedimensional magnetic field is obtained from a potential extrapolation of the filtered LOS photospheric magnetogram measured at 16:00 UT using the method described in Otto et al. (2007).

\section{Results and discussion}

In addition to the pattern used in Santos \& Büchner (2007), we have performed two more simulation runs using the velocity patterns shown in Fig. 3. The simulation starts always from the same initial configuration (the one used in Santos \& Büchner 2007). The only difference between the simulation runs is the velocity pattern used as boundary condition to perturb the system. This allows to investigate the influence of the velocity patterns on the formation and location of current sheets.

Strong electric currents are expected to develop in and around regions where the magnetic field strongly changes the connectivity, i.e. at so called quasi-separatrix layers (Priest \& Démoulin 1995; Démoulin et al. 1996a,b). It is expected that the plasma motion will affect the generation of electric currents in these regions (Aulanier et al. 2005; Büchner 2006). The position of the footpoints of the quasi-separatrix field lines can be 

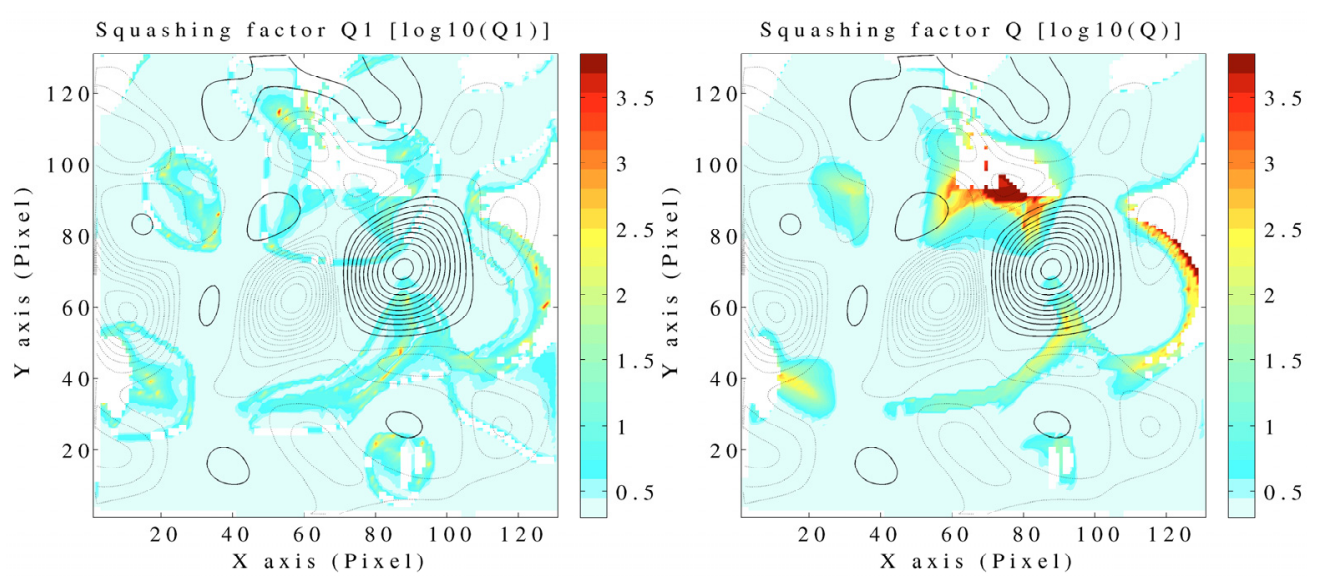

Fig. 4. Squashing factor values (colour code) obtained for a potential field extrapolation of the photospheric LOS component measured below an EUV BP on 2006 Jan. 19, at 16:00 UT. On the left the squashing factor is shown, calculated considering only the position where the footpoints of the field lines are mapped $\left(Q_{1}\right)$. On the right the squashing factor is calculated considering also the ratio of the absolute value of the normal component of the magnetic field in the two magnetic conjugate points $(Q)$. The contour lines show the position of the negative (thin line) and positive (thick line) magnetic polarities. estimated by the computation of a parameter called squashing factor (Titov et al. 2002). The footpoints of the quasi-separatrix layers are supposed to be located in regions where the values of the squashing factor is large. The squashing factor was calculated for the EUV BP observed on 2006 Jan. 19 considering the magnetic field obtained from the potential extrapolation of the LOS component of the photospheric field measured at 16:00 UT of that day (see Büchner 2006 for details of our calculation). Figure 4 shows the squashing factor calculated considering only the relation between the positions where the footpoints of the field lines are mapped $\left(Q_{1}\right.$, left panel) and considering the ratio of the absolute value of the normal component of the magnetic field in the two magnetic conjugated points $(Q$, right panel). The results obtained using the two different methods are similar and show well defined regions where the values of the squashing factor are large. The contour lines in Fig. 4 show also the position of the positive (thick line) and the negative (thin line) magnetic polarities. Due to the plasma motion through strong magnetic field gradients below the transition region, it is expected that the strongest electric current densities will develop at lower heights in the solar atmosphere.

Indeed, all the different patterns of photospheric plasma motion used as boundary condition for the model give rise to electric currents located mainly below the transition region, just above the main polarities of the magnetic field. We consider the current components parallel $\left(j_{\|}\right)$and perpendicular $\left(j_{\perp}\right)$ to the magnetic field separately, and analyze them independently. The results obtained using the velocity pattern responsible for the evolution between 16:00 UT and 16:30 UT were already presented in Santos \& Büchner (2007). Figure 3 of Santos \& Büchner (2007) shows the isosurfaces of a parallel current $j_{\|}=$ $2 j_{0}$, where $j_{0} \approx 1.59 \times 10^{-4} \mathrm{~A} / \mathrm{m}^{2}$ is the value used to normalize the electric current. This current system results from the application of a velocity pattern which moves the plasma above the negative polarity region southward, away from the positive polarity region. This motion stretches the magnetic flux tubes, by increasing their magnetic energy contents. The horizontal motion also gives rise to electric currents perpendicular to the magnetic field. Figure 4 of Santos \& Büchner (2007) shows the isosurfaces of a perpendicular current $j_{\perp}=2 j_{0}$. The parallel and perpendicular currents are formed in the chromosphere, transition region and lower corona. The vertical profile of the squared current density integrated over the area $A$, where $A$ is the total area of the horizontal plane $x-y$ in the simulation box,

$W_{j}(z)=\int_{A} j^{2} \mathrm{~d} x \mathrm{~d} y$

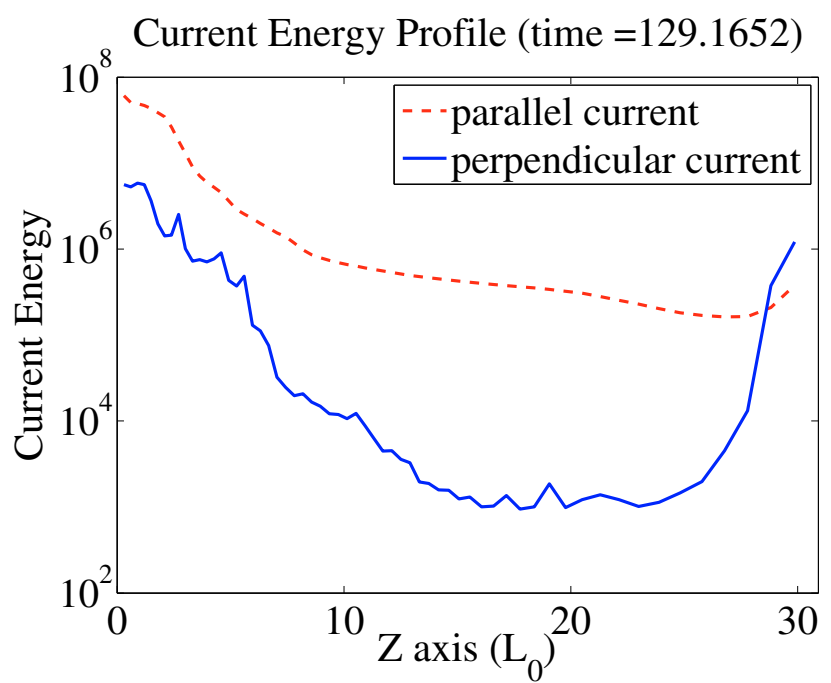

Fig. 5. Height profile of the electric current energy obtained by Santos \& Büchner (2007), calculated considering the element of area in physical units.

is calculated at $t=1300 \mathrm{~s}$ for the parallel and the perpendicular currents. It is used as a proxy for the current energy assuming a constant resistivity. We present again the results for the case of Santos \& Büchner (2007) calculated considering the element of area in physical units (Fig. 5), for a better comparison with the results obtained here. The electric current energy $W_{j}$ is minimum between $z=15 L_{0}$ and $z=25 L_{0}$, where $L_{0}=5 \times 10^{5} \mathrm{~m}$ is the value used as the characteristic length scale. The highest values for the electric current energy are found for $z<15 L_{0}$ and $z>$ $25 L_{0}$. The model transition region corresponds to $z \approx 5 L_{0}$. The increase of the energy near the top of the simulation box is a boundary effect of the simulation.

The results of the two velocity patterns obtained by LCT for later times of the evolution of the EUV BP (16:30 UT17:30 UT) are presented here, in complement to the results presented in Santos \& Büchner (2007). During the interval 16:30 UT-17:00 UT (left panel of Fig. 3) the negative polarity moves northward, in the direction toward the positive polarity region. This leads to a decrease of the total magnetic flux (flux cancelation). The electric currents resulting from this motion are shown in Figs. 6 and 7. The parallel and perpendicular currents are formed preferentially below $z=10 L_{0}$. However, parallel currents start to develop higher in the solar atmosphere $\left(z \approx 20 L_{0}\right)$ due to the interaction of the magnetic fields resulting from the 

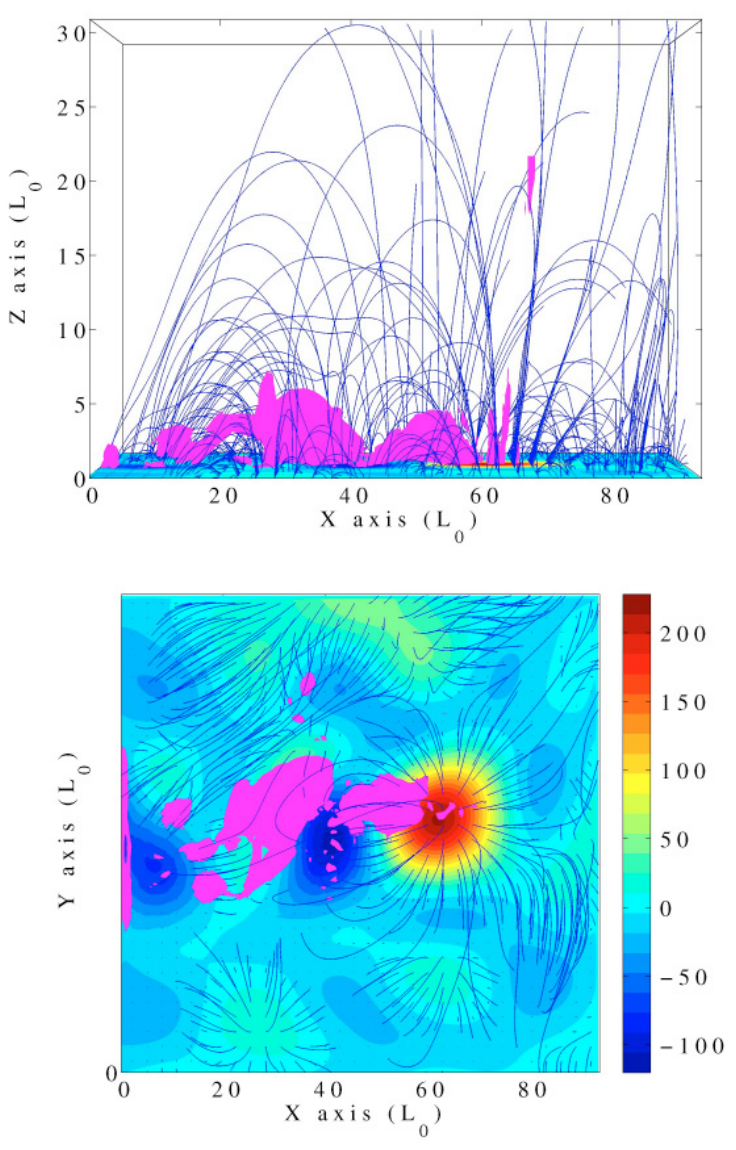

Fig. 6. Lateral view (top panel) and top view (bottom panel) of the isosurfaces of a parallel current $j_{\|}=2 j_{0}$ at the instant $t=1300 \mathrm{~s}$, resulting from the application of the velocity pattern shown in the left panel of Fig. 3 as boundary condition of the model. The colour code shows the vertical component of the photospheric magnetic field, with the magnetic field values given in Gauss, and the lines correspond to the magnetic field lines of force. The isosurfaces of parallel current are shown in magenta.

converging motion of the two opposite magnetic field polarities that form the bipole. Note that the parallel currents are by an order of magnitude stronger than the perpendicular ones as previously shown in Büchner (2006). The profile of the electric current energy (Fig. 8) shows that the highest values for the electric current energy are found for $z<10 L_{0}$, lower than in the case studied in Santos \& Büchner (2007). However, the electric current energy above $z \approx 15 L_{0}$ is higher than the one obtained for the evolution after 16:00 UT. This is also a consequence of the interaction of the magnetic fields resulting from the converging motion of the two opposite magnetic field polarities that form the bipole. The energy associated with the parallel current dominates over that associated with the perpendicular current, i.e. the field stays basically force-free.

During the interval 17:00 UT-17:30 UT (right panel of Fig. 3) the negative flux concentration brakes apart. A part of it is moving southward and the other part moves northward in the direction toward the positive polarity. The isosurfaces of current obtained using this velocity pattern as boundary condition are shown in Figs. 9 and 10. Figure 11 shows the electric current energy profile for this period. Regions of minimum and higher values for the electric current energy are similar to that obtained in Santos \& Büchner (2007). However, the electric current energy is lower than in the other two cases in the region below
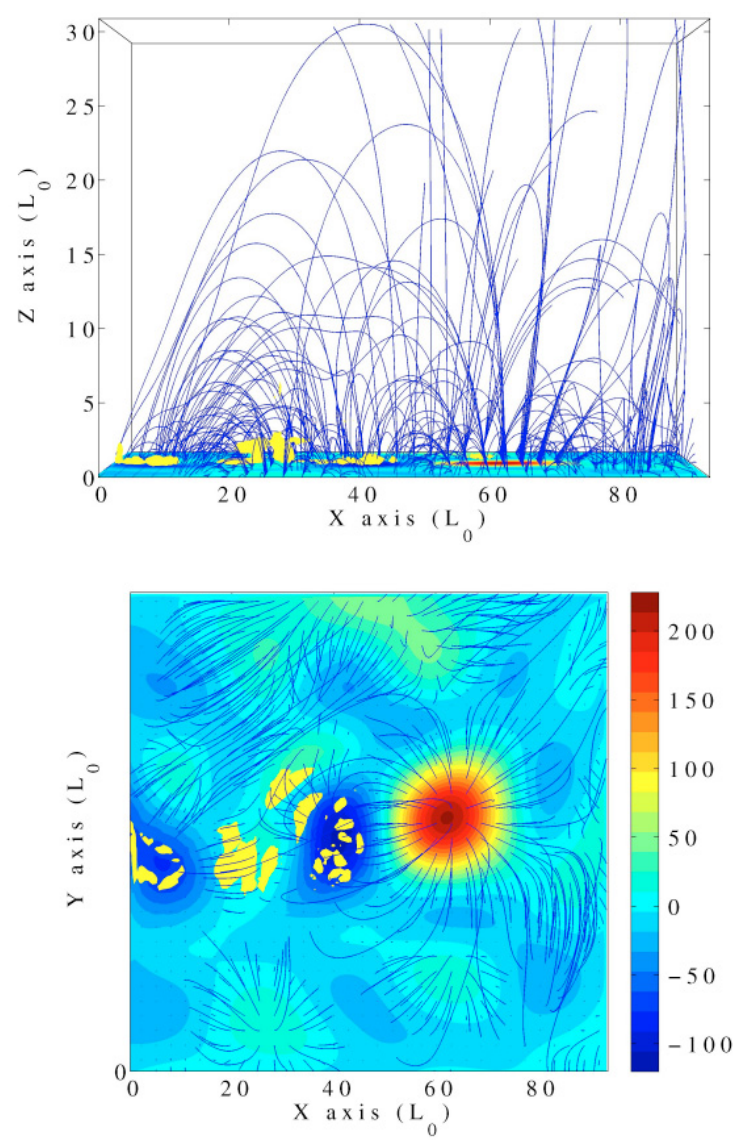

Fig. 7. Lateral view (top panel) and top view (bottom panel) of the isosurfaces of a perpendicular current $j_{\perp}=2 j_{0}$ at the instant $t=1300 \mathrm{~s}$, resulting from the application of the velocity pattern shown in the left panel of Fig. 3 as boundary condition of the model. The colour code shows the vertical component of the photospheric magnetic field, with the magnetic field values given in Gauss, and the lines correspond to the magnetic field lines of force. The isosurfaces of perpendicular current are shown in yellow.

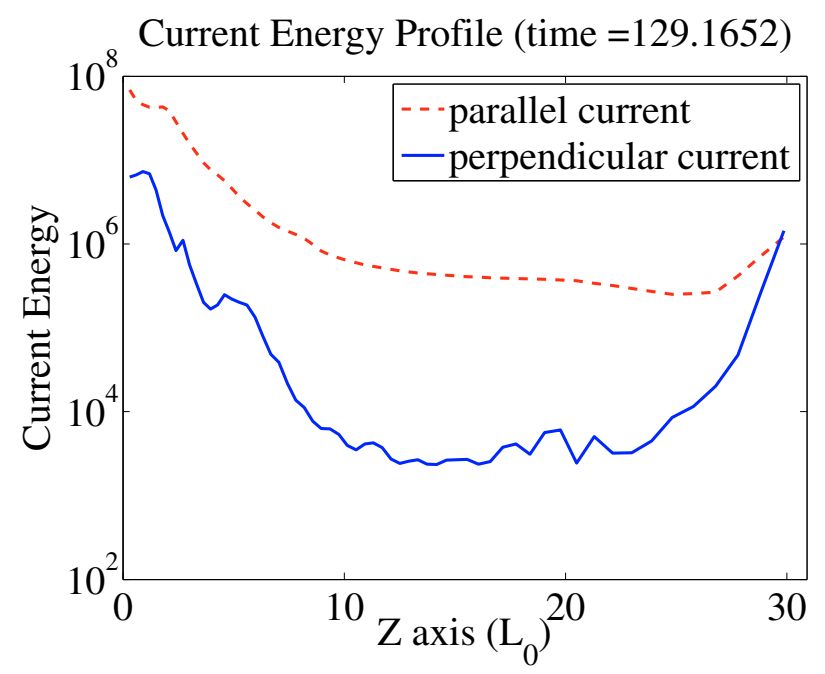

Fig. 8. Height profile of the electric current energy obtained by applying the velocity pattern shown in the left panel of Fig. 3 as boundary condition of the model at $t=1300 \mathrm{~s}$.

$z=10 L_{0}$. The reason is probably the smaller amplitudes of the velocities used in this run. As in the previous cases, the isosurfaces of current appear preferentially below $z=10 L_{0}$ and the 

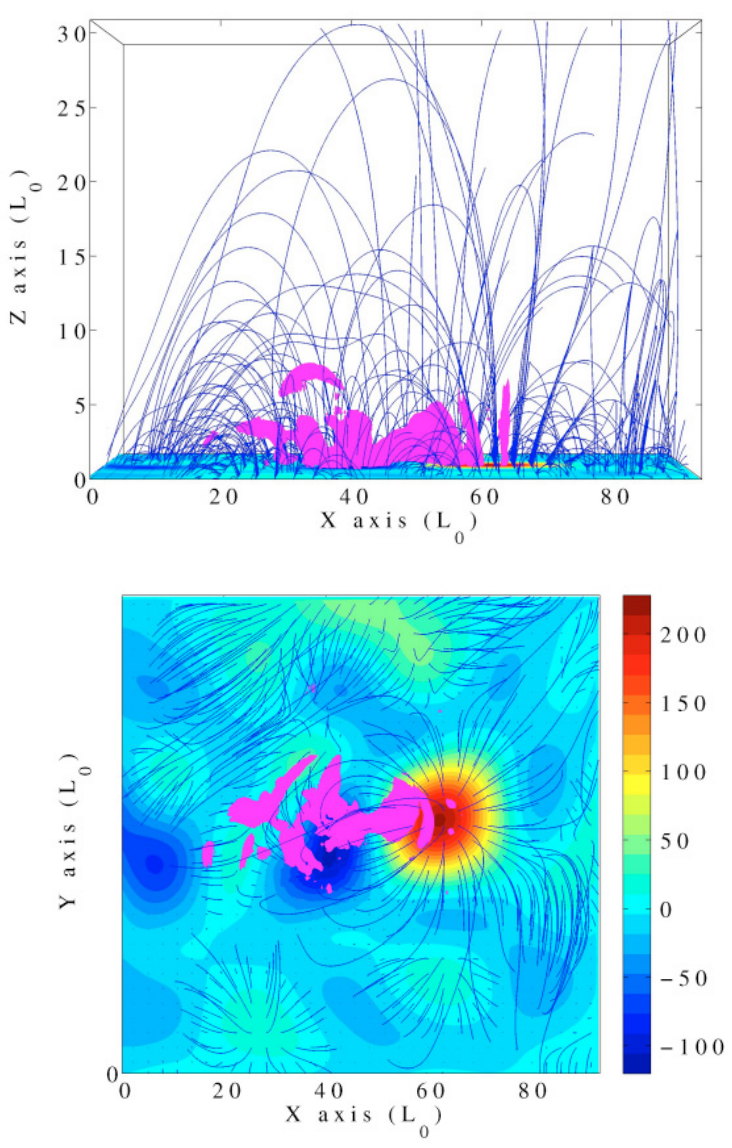

Fig. 9. Lateral view (top panel) and top view (bottom panel) of the isosurfaces of a parallel current $j_{\|}=2 j_{0}$ at the instant $t=1300 \mathrm{~s}$, resulting from the application of the velocity pattern shown in the right panel of Fig. 3 as boundary condition of the model. The colour code shows the vertical component of the photospheric magnetic field, with the magnetic field values given in Gauss, and the lines correspond to the magnetic field lines of force. The isosurfaces of parallel current are shown in magenta.

parallel current is more distributed than the perpendicular current.

Figure 12 presents the values of the parallel and perpendicular current densities integrated along the magnetic field for the three different runs. The currents develop in approximately the same region, independently of the motion pattern used as boundary condition. A comparison with Fig. 4 shows that these regions coincide with the regions where the values of the squashing factor are large. This confirms the conjecture that strongest currents are expected to develop in and around regions where the magnetic field strongly change the connectivity (quasi-separatrix layers).

We also calculate the evolution in time of the total magnetic energy inside the simulation box

$W_{B}=\int_{V} \frac{B^{2}}{2 \mu_{0}} \mathrm{~d} V$

Figure 13 shows the evolution of the total magnetic energy $\left(W_{B}\right)$ versus time. The different runs correspond to the application of the different velocity patterns as boundary condition. In the first case, for the evolution after 16:00 UT (Santos \& Büchner 2007), the total magnetic energy increases while the opposite magnetic polarities move apart. The second case, for the evolution after 16:30 UT, represents the effect of flux cancelation: a decrease
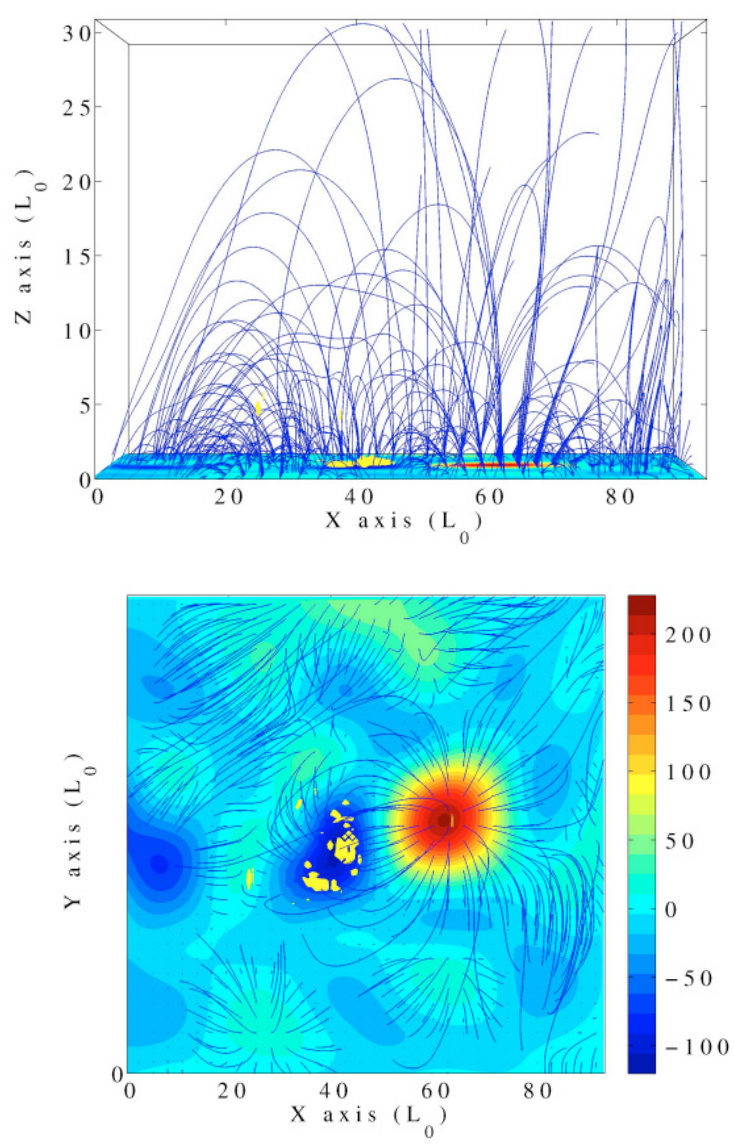

Fig. 10. Lateral view (top panel) and top view (bottom panel) of the isosurfaces of a perpendicular current $j_{\perp}=2 j_{0}$ at the instant $t=1300 \mathrm{~s}$, resulting from the application of the velocity pattern shown in the right panel of Fig. 3 as boundary condition of the model. The colour code shows the vertical component of the photospheric magnetic field, with the magnetic field values given in Gauss, and the lines correspond to the magnetic field lines of force. The isosurfaces of perpendicular current are shown in yellow.

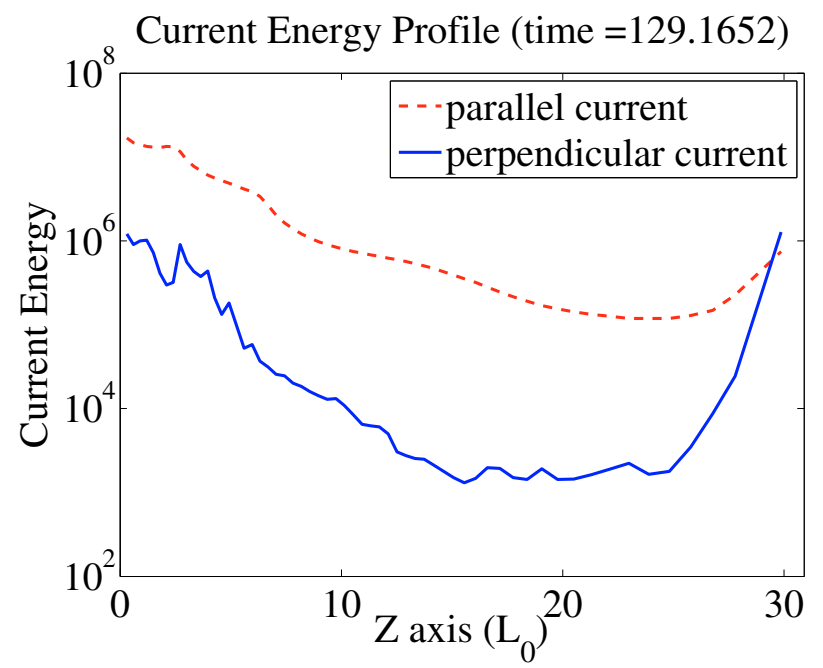

Fig. 11. Height profile of the electric current energy obtained by applying the velocity pattern shown in the right panel of Fig. 3 as boundary condition of the model at $t=1300 \mathrm{~s}$.

of the total magnetic energy of the system. The third case, describing the evolution after 17:00 UT, is intermediate between case 1 and case 2 . It is characterized by a break off of the negative 

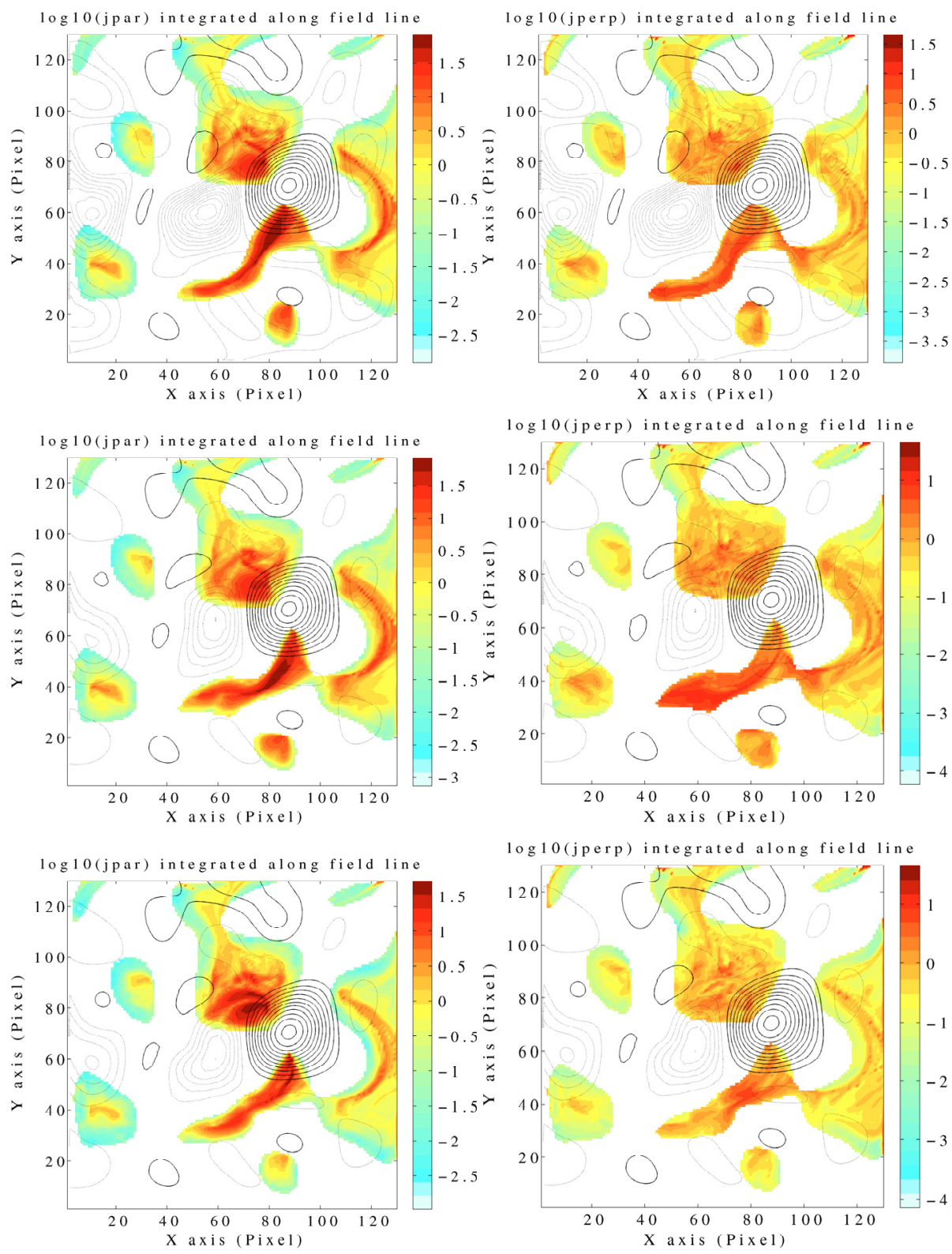

Fig. 12. Parallel (left) and perpendicular (right) current density integrated along the magnetic field for the three different runs: 16:00 UT16:30 UT (top), 16:30 UT-17:00 UT (middle) and 17:00 UT-17:30 UT (bottom).

polarity. This is followed by the motion of part of it away from the positive polarity and of another part of it in direction to the positive polarity which maintains the total magnetic energy almost constant.

\section{Conclusions}

1. The application of LCT to the photospheric magnetic field during the interval 16:00 UT-17:30 UT revealed three different patterns of the plasma motion near the negative magnetic polarity: shearing, convergence and fragmentation.

2. All three patterns of horizontal motion of the photospheric plasma give rise to electric currents, mainly in the chromosphere, but also in the transition region and lower corona $(z \leq 7.5 \mathrm{Mm})$.

3. Currents do not develop everywhere where the plasma motion is applied but only in places where at the same time the magnetic field connectivity changes significantly (quasiseparatrix layers).
4. The main result of this work is the fact that the electric currents develop in approximately the same regions, independent on the specific form of the plasma motion applied.

5. A comparison with TRACE and EIT data has shown that regions of enhanced currents coincide well with the area where the EUV BP is located. With this result the restriction to emerging and canceling patterns of motion that was present in the previous models disappears. As observed by Brown et al. (2001), different kinds of motion of the magnetic structures can be associated to the BP.

6. The EIT $195 \AA$ and TRACE $1550 \AA$ images show that the $\mathrm{BP}$ consists of bright kernel surrounded by a fainter bright region. The position of the kernel changes with time, while the fainter bright region seems to be located always over the same area. If the mechanism of heating by parallel DC current dissipation is efficient, as suggested by Büchner \& Elkina $(2005,2006)$, these currents could explain the appearance of the bright point in the EIT data. The change in the position of the kernel would be associated to small changes in the position of the electric currents caused by the change in the plasma velocity pattern. 
Total Magnetic Energy

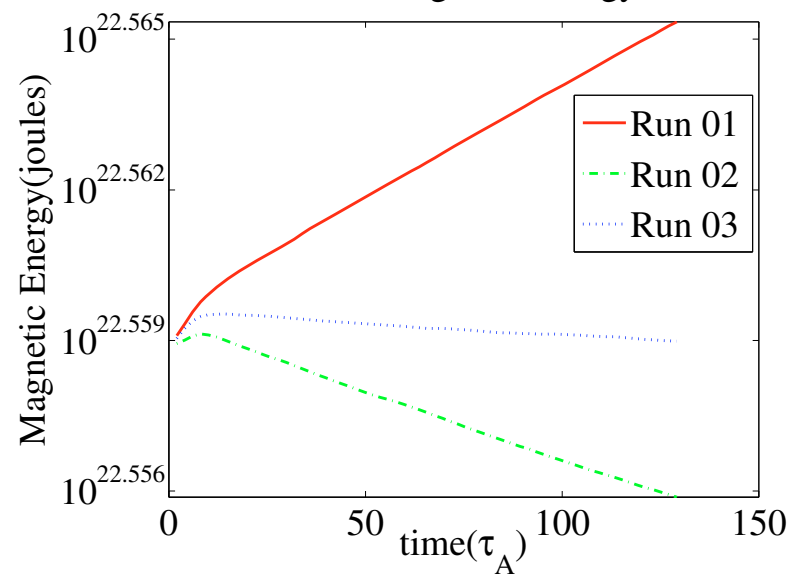

Fig. 13. Total magnetic energy (joules) versus time $\left(\tau_{\mathrm{A}}\right)$. The different runs correspond to the application of the different velocity patterns as a boundary condition to the model. The Alfén time $\left(\tau_{\mathrm{A}}\right)$ is equal to $10 \mathrm{~s}$.

Acknowledgements. The authors would like to acknowledge the ISSI team lead by G. Poletto on "Role of current sheets in solar eruptive events" and the Ph.D. fellowship of J. Santos at the International Max-Planck Research School-IMPRS on Solar System Research which supported this work

\section{References}

Alfvén, H. 1947, MNRAS, 107, 211

Aulanier, G., Pariat, E., \& Démoulin, P. 2005, A\&A, 444, 961
Birn, J., \& Priest, E. R. 2007, Reconnection of magnetic fields: magnetohydrodynamics and collisionless theory and observations (Reconnection of magnetic fields: magnetohydrodynamics and collisionless theory and observations, ed J. Birn \& E. R. Priest (Cambridge: Cambridge University Press), ISBN: 9780521854207 (hbk.))

Brown, D. S., Parnell, C. E., Deluca, E. E., Golub, L., \& McMullen, R. A. 2001, Sol. Phys., 201, 305

Büchner, J. 2006, Space Sci. Rev., 122, 149

Büchner, J., \& Elkina, N. 2005, Space Sci. Rev., 121, 237

Büchner, J., \& Elkina, N. 2006, Phys. Plasmas, 13, 2304

Büchner, J., Nikutowski, B., \& Otto, A. 2004a, in SOHO 15 Coronal Heating, ed. R. W. Walsh, J. Ireland, D. Danesy, \& B. Fleck, ESA SP, 575, 23

Büchner, J., Nikutowski, B., \& Otto, A. 2004b, in Multi-Wavelength Investigations of Solar Activity, ed. A. V. Stepanov, E. E. Benevolenskaya, \& A. G. Kosovichev, IAU Symp., 223, 353

Démoulin, P., \& Berger, M. A. 2003, Sol. Phys., 215, 203

Démoulin, P., Henoux, J. C., Priest, E. R., \& Mandrini, C. H. 1996a, A\&A, 308, 643

Démoulin, P., Priest, E. R., \& Lonie, D. P. 1996b, J. Geophys. Res., 101, 7631

Golub, L. 1980, Roy. Soc. London Philos. Trans. Ser. A, 297, 595

Habbal, S. R. 1992, Ann. Geophys., 10, 34

Longcope, D. W. 1998, ApJ, 507, 433

November, L. J., \& Simon, G. W. 1988, ApJ, 333, 427

Otto, A., Büchner, J., \& Nikutowski, B. 2007, A\&A, 468, 313

Parker, E. N. 1972, ApJ, 174, 499

Parnell, C. E., Priest, E. R., \& Titov, V. S. 1994, Sol. Phys., 153, 217

Priest, E. R., \& Démoulin, P. 1995, J. Geophys. Res., 100, 23443

Priest, E. R., Parnell, C. E., \& Martin, S. F. 1994, ApJ, 427, 459

Santos, J. C., \& Büchner, J. 2007, Astrophys. Space Sci. Trans., 3, 29

Titov, V. S., Hornig, G., \& Démoulin, P. 2002, J. Geophys. Res. (Space Physics), 107,1164

Tur, T. J., \& Priest, E. R. 1976, Sol. Phys., 48, 89

Webb, D. F. 1986, in Coronal and Prominence Plasmas, ed. A. I. Poland, 329 